LESSON OF THE MONTH

\title{
Differential diagnosis of calf pain with musculoskeletal ultrasound imaging
}

D Kane, P V Balint, R Gibney, B Bresnihan, R D Sturrock

Series editor: Anthony D Woolf

Ann Rheum Dis 2004;63:11-14. doi: 10.1136/ard.2002.002824

\section{CASE HISTORIES}

Case 1: Massive Baker's cyst presenting as a deep venous thrombosis (fig 1)

A 68 year old woman presented to the accident and emergency department describing a three day history of acute left calf pain and swelling associated with severe difficulty in weight bearing. She had been previously diagnosed with hypertension, peptic ulcer disease, and had had seronegative rheumatoid arthritis affecting hands, knees, and hips for the past five years. Regular drugs included celecoxib and ranitidine. She was a smoker with a body mass index of 27 and had been on an airline flight from Spain to Scotland two weeks previously. The D-dimer level was increased at $1463 \mu \mathrm{g} / \mathrm{l}$ and she was initially treated with Dalteparin for a presumptive diagnosis of deep venous thrombosis (DVT). A Duplex ultrasound (US) scan of the leg was performed and did not show any evidence of a DVT. The left calf pain had now increased and new bruising was noticed at the inferoposterior aspect of the left calf. Dalteparin was discontinued and musculoskeletal ultrasound (MSUS) confirmed the presence of a large Baker's cyst extending from the popliteal fossa to the junction of the gastrocnemius and Achilles tendon, at the site of the new bruising (fig 1). The patient now admitted that calf pain and swelling had been present for several months. A diagnosis of Baker's cyst with minor rupture was made. The left knee was aspirated and injected with little improvement in calf swelling. Two attempts to reduce the cyst by direct US guided aspiration with an $18 \mathrm{G}$ and $16 \mathrm{G}$ needle were unsuccessful. The patient declined a further surgical opinion and was managed conservatively.

Case 2: Achilles pain in ankylosing spondylitis (fig 2) A 50 year old man presented with an exacerbation of longstanding ankylosing spondylitis affecting cervical and lumbar spine, both hips, and both ankles and heels. He had noted a recent spontaneous increase in left lower calf and heel pain that was beginning to resolve. On clinical examination he was found to have tenderness in both Achilles tendons-left worse than right—consistent with a diagnosis of bilateral Achilles tendonitis. A US examination was performed to confirm the diagnosis. The right Achilles tendon was normal. At the middle third of the left Achilles tendon a $2 \mathrm{~cm} \times 0.25 \mathrm{~cm} \times 0.36 \mathrm{~cm}$ anechoic collection was observed within the Achilles tendon (fig 2). A small area of disruption between the fibres of the deep part of the Achilles tendon was noted adjacent to the collection. The findings were consistent with a left Achilles tendon cystic fluid collection resulting from an atraumatic longitudinal Achilles tendon tear. No evidence of retrocalcaneal bursitis was found, and the Achilles pain was managed conservatively.

Case 3: Diagnosis and treatment of "tennis leg" with ultrasound imaging (fig 3)

A 51 year old man in good health presented with diffuse swelling and tenderness of the right calf, worse on weight bearing. This had developed acutely during a tennis match, but had worsened over four days. He was due to undertake a long haul flight and wanted a musculoskeletal injury to be confirmed and a DVT excluded. A US study of the calf demonstrated a well localised hypoechoic collection interposed between the medial head of the gastrocnemius and the soleus muscle, typical of a small tear of the medial head of gastrocnemius or of the plantaris tendon. About $30 \mathrm{ml}$ of serosanguinous fluid was drained under US guidance, resulting in collapse of the central hypoechoic fluid collection and restoration of the normal apposition of the gastrocnemius and soleus muscles. The patient noted a marked symptomatic and functional improvement immediately after the procedure.

\section{DISCUSSION}

The differential diagnosis of calf pain and swelling includes DVT, cellulitis, Baker's cyst, muscular injury, tumour or infection, arterial aneurysm, and Achilles tendon inflammation or rupture. Much emphasis in current clinical practice is placed on the diagnosis of DVT owing to the serious risk of pulmonary embolism. ${ }^{1}$ The sophisticated use of risk stratification models, D-dimer measurement, and Duplex venous studies correctly identifies most DVTs. However, a number of musculoskeletal disorders may present with a similar clinical picture and require careful evaluation in order to avoid inappropriate investigation and management. Dual pathology of a DVT and Baker's cyst has been reported in 3\% of DVTs, and the compressive effects of the cyst may predispose to the development of a DVT. ${ }^{2}$ Additionally, D-dimer levels may be raised in patients with inflammatory arthritis, ${ }^{3}$ leading to a false positive result and unnecessary investigations and treatment. Although dual pathology or the presentation of a Baker's cyst mimicking a DVT is well described, it is often not considered or diagnosed during the initial venous US study. We suggest that both venous and musculoskeletal US scanning should be performed in cases of acute calf pain and swelling and in all cases of calf pain with a negative venous US examination.

In the three cases described, a careful history was the key to the identification of a probable underlying musculoskeletal disorder. In the first case the patient presented to casualty and gave a history of acute calf pain and swelling. D-dimer levels were raised and treatment was started with low molecular weight heparin. Her symptoms subsequently worsened with a further leak of the Baker's cyst. On further questioning, she admitted to a much longer history of calf swelling, and MSUS confirmed a massive Baker's cyst. In the second case the patient clearly identified a recent atraumatic

\footnotetext{
Abbreviations: $\mathrm{CT}$, computed tomography; DVT, deep venous thrombosis; MRI, magnetic resonance imaging; MSUS, musculoskeletal ultrasound; US, ultrasound
} 


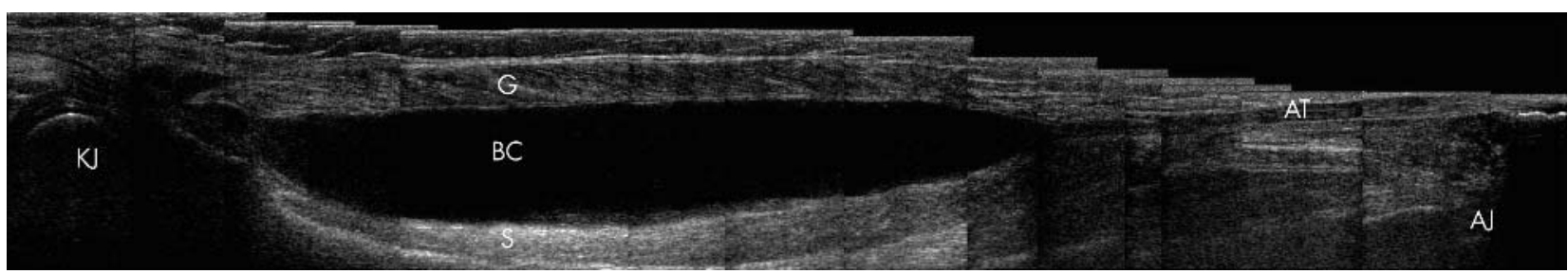

Figure 1 Baker's cyst (photomontage). Photomontage longitudinal image of the calf: Hypoechoic effusion is seen immediately posterior to the knee joint (KJ). This initially extends superficially to the medial head of the gastrocnemius (G) and then dissects between the gastrocnemius and the soleus (S) towards the ankle joint (AJ) for the proximal two thirds of the calf, ending proximal to the formation of the Achilles tendon (AT) by the soleus and gastrocnemius.

exacerbation of calf pain on a background of longstanding Achilles enthesopathy. MSUS was performed to see if the recent worsening of symptoms was due to the development of retrocalcaneal bursitis that would have been amenable to corticosteroid injection. In the third case the sudden onset of pain while engaged in a non-contact activity suggested a diagnosis of muscular injury. The subsequent development of swelling with a planned long haul flight led to the need to confirm the diagnosis by MSUS.

Clinical examination in all three patients allowed localisation of tenderness and swelling, but in all three cases MSUS imaging gave a rapid, anatomical confirmation of the diagnosis. MSUS is increasing in use in rheumatological practice because it allows detailed anatomical evaluation of joints and surrounding soft tissues. It is safe (no ionising radiation), reproducible, portable, inexpensive by comparison with computed tomography (CT) and magnetic resonance imaging (MRI) scanning and may be performed rapidly by the experienced examiner. ${ }^{4}$ The application of MSUS has been clearly shown to be better than the detection on clinical examination of knee effusion and popliteal cyst in rheumatoid arthritis, ${ }^{6-8}$ better for the detection of enthesopathy ${ }^{9}$ in the leg, and better for the localisation of effusions for aspiration. ${ }^{10}$

Baker's cyst (enlargement of the gastrocnemiosemimembranosus bursa) is a common cause of calf swelling and may be confirmed by orthography, ultrasound, CT scanning or MRI. MSUS is the preferred investigation as it is inexpensive, sensitive, and widely available. Asymptomatic cysts found incidentally do not require treatment. Symptoms usually develop when a cyst enlarges or leaks into the calf, producing swelling and pain. Specific treatment with intraarticular knee injection may relieve symptoms if a knee effusion is present, thus reducing the production of synovial fluid in the knee and subsequent filling into the Baker's cyst. Direct aspiration of a Baker's cyst may be performed when it produces significant local pressure and discomfort. In cases of large or recalcitrant cysts, surgical excision of the cyst and its connection to the joint produces relief of symptoms, but failure to treat associated knee inflammation adequately may lead to a high rate of recurrence. ${ }^{11}$

In inflammatory arthritis, Achilles tendonitis is often associated with retrocalcaneal bursitis, ${ }^{12}{ }^{13}$ which responds to corticosteroid injection of the bursa under MSUS guidance. ${ }^{14}$ Examination of the Achilles tendon insertion underestimates the presence of retrocalcaneal bursitis as compared with MSUS. In the second case we sought to determine if the recent increase in symptoms related to a bursitis but instead found evidence of a previous minor Achilles tendon rupture with associated fluid collection. As injection of corticosteroid adjacent to the Achilles tendon may be associated with a further softening of the Achilles tendon and possible rupture, we chose not to proceed with bursal injection. ${ }^{15} 16$

Complete or partial rupture of the fibres of the musculotendinous junction of the medial head of the gastrocnemius muscle, also called "tennis leg", is one of the most common muscle injuries in men older than 40 years who participate in racquet sports, alpine skiing, and running. ${ }^{17}$ It causes acute leg swelling and tenderness with significant functional impairment, but usually resolves after a few weeks with a low recurrence rate. MSUS allows for rapid confirmation of the clinical diagnosis ${ }^{17} 18$ and exclusion of other disease. Partial muscle tears are seen on US as either small $(<2 \mathrm{~cm})$, heterogeneous, echogenic areas or focal hypoechoic change within the muscle fibres, whereas complete tears are larger and are often associated with a hypoechoic haematoma. A rupture of the plantaris tendon may also present with a similar clinical picture and may even coexist with a gastrocnemius tear. US features are similar, though the fluid collection associated with a plantaris tendon tear may be more proximal and the ruptured plantaris tendon may be
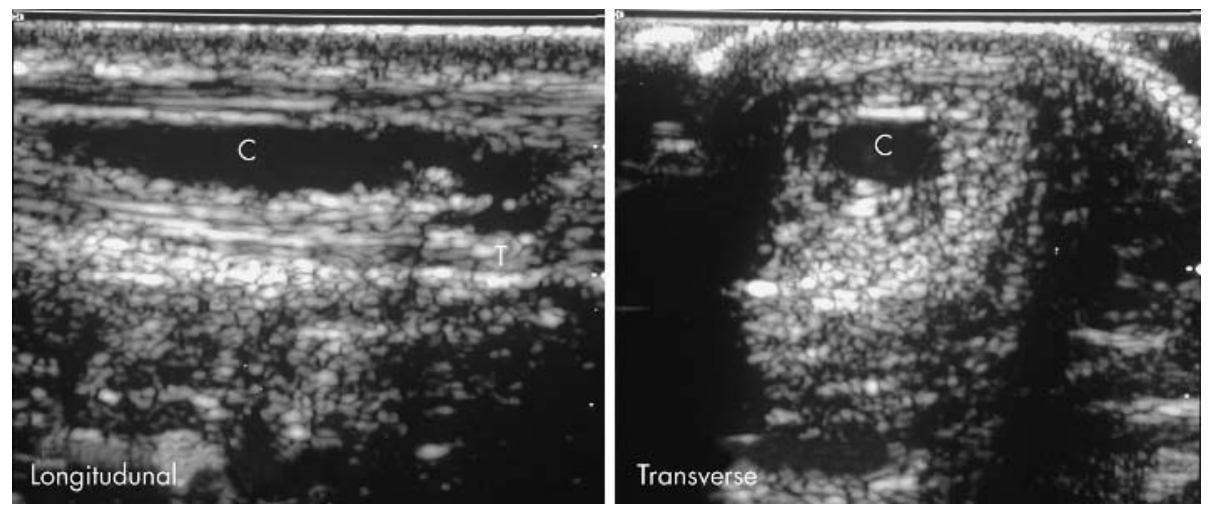

Figure 2 Achilles tendon tear. (A) Longitudinal and (B) transverse images of the calf: at the middle third of the left Achilles tendon there is an anechoic collection (C) lying within the Achilles tendon. A small area of disruption (T) of the deep part of the Achilles tendon fibrillar structure is adjacent to the collection. The findings are consistent with a left Achilles tendon fluid collection resulting from an atraumatic partial Achilles tendon tear (T). 

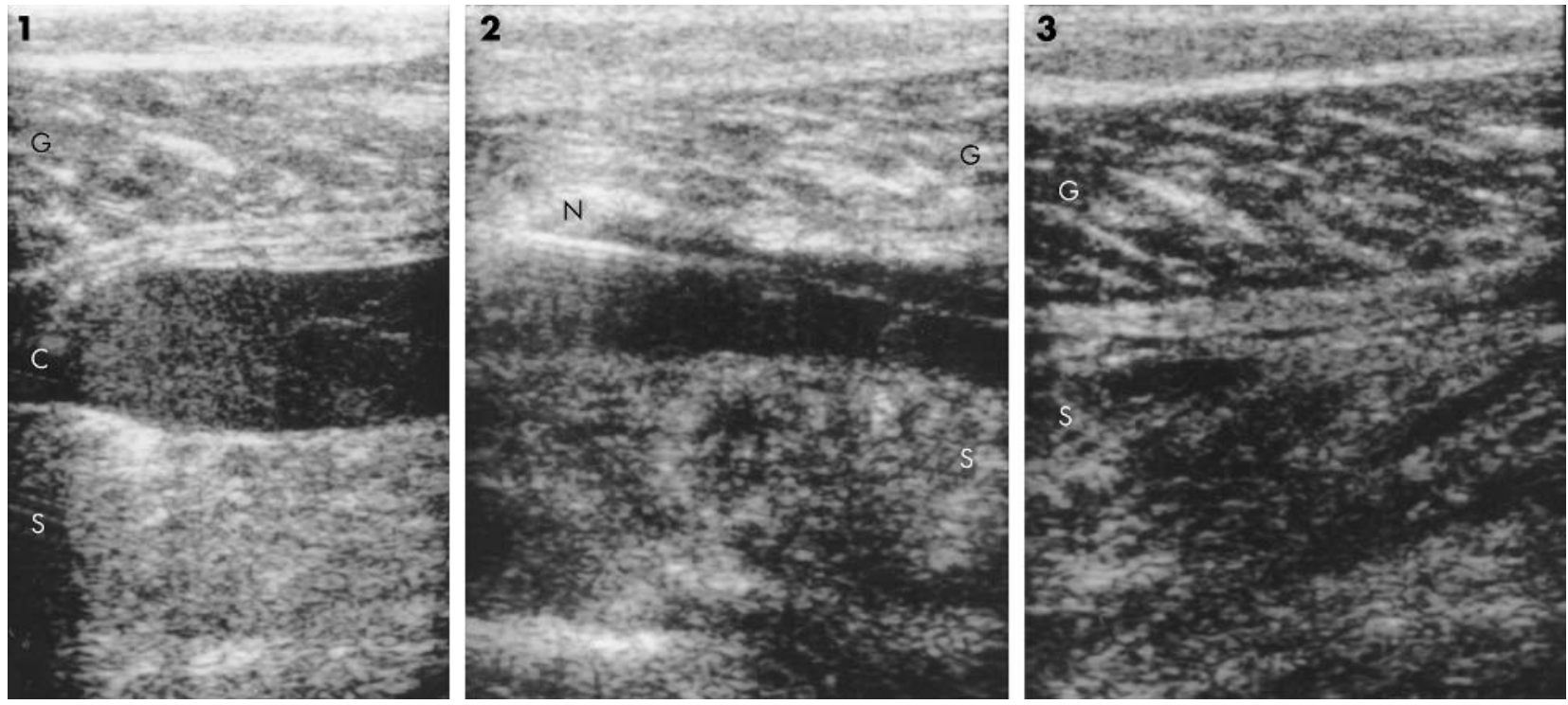

Figure 3 "Tennis Leg". Serial Longitudinal images of the calf: (1) There is a well localised hypoechoic collection (C) interposed between the medial head of the gastrocnemius (G) and the soleus muscle (S), typical of a tear. (2) About $30 \mathrm{ml}$ of blood stained fluid was drained with a $21 \mathrm{G}$ needle (N) under ultrasound guidance. (3) This resulted in the collapse of the central hypoechoic fluid collection with restoration of the normal apposition of the gastrocnemius and soleus muscles.

visualised. US is an inexpensive, safe, and quick diagnostic test in suspected tennis leg. When a haematoma or fluid collection is present, US guided drainage may produce a rapid relief of symptoms and restoration of function.

MSUS improves the success rate of joint aspiration in everyday rheumatology practice. ${ }^{10}$ In the first case, aspiration using real time US imaging was unsuccessful, possibly because the collection was long standing and gelatinous. The hypoechoic nature of the cyst was more typical for an effusion, and further confirmation of a gelatinous cyst at surgery was not possible. In the third case, US guided aspiration of the blood stained collection was successful and produced a prompt symptomatic response. MSUS has the potential to significantly improve clinical diagnosis and management of patients presenting with calf pain. Rheumatologists are increasingly using MSUS in their daily practice to aid diagnosis and therapeutic skills. Initial evidence suggests that the application of MSUS in rheumatology improves diagnostic and therapeutic skills and has a significant impact on patient management. ${ }^{19}$

\section{THE LESSONS}

- Careful evaluation of the history usually indicates a musculoskeletal cause of calf pain.

- D-dimers may be raised in patients with inflammatory arthritis in the absence of a DVT.

- The presentation of a Baker's cyst mimicking a DVT is well described; we recommend musculoskeletal ultrasound in all cases of calf pain and swelling with a negative venous ultrasound study of the lower limbs.

- Musculoskeletal ultrasound allows differentiation of synovial fluid cysts or collections from muscle haematoma and tears aiding rapid differential diagnosis of a number of causes of musculoskeletal calf pain.

- Musculoskeletal ultrasound allows guidance of injection therapy in the management of calf pain.

\section{Authors' affiliations}

D Kane, R D Sturrock, Centre for Rheumatic Diseases, University

Department of Medicine, Glasgow Royal Infirmary, Glasgow, UK
P V Balint, 3rd Rheumatology Department, National Institute of Rheumatology and Physiotherapy, Budapest, Hungary

R Gibney, B Bresnihan, Department of Diagnostic Imaging, St Vincent's University Hospital, Dublin 4, Ireland

Correspondence to: Dr D Kane, Centre for Rheumatic Diseases, University Department of Medicine, Glasgow Royal Infirmary, 10 Alexandra Parade, Glasgow G31 2ER, Scotland, UK; dk44a@clinmed.gla.ac.uk

Accepted 15 May 2003

\section{REFERENCES}

1 Janssen MC, Wollersheim H, Verbruggen B, Novakova IR. Rapid D-dimer assays to exclude deep venous thrombosis and pulmonary embolism: current status and new developments. Semin Thromb Hemost 1998;24:393-400.

2 Langsfeld M, Matteson B, Johnson W, Wascher D, Goodnough J, Weinstein E. Baker's cysts mimicking the symptoms of deep vein thrombosis: diagnosis with venous duplex scanning. J Vasc Surg 1997;25:658-62.

3 McEntegart A, Capell HA, Creran D, Rumley A, Woodward M, Lowe GD. Cardiovascular risk factors, including thrombotic variables, in a population with rheumatoid arthritis. Rheumatology (Oxford) 2001;40:640-4.

4 Balint PV, Sturrock RD. Intraobserver repeatability and interobserver reproducibility in musculoskeletal ultrasound imaging measurements. Clin Exp Rheumatol 2001;19:89-92.

5 Wakefield RJ, Gibbon WW, Emery P. The current status of ultrasonography in rheumatology. Rheumatology (Oxford) 1999;38:195-8.

6 Fam AG, Wilson SR, Holmberg S. Ultrasound evaluation of popliteal cysts on osteoarthritis of the knee. J Rheumatol 1982:9:428-34.

7 Hauzeur JP, Mathy L, De Maertelaer V. Comparison between clinical evaluation and ultrasonography in detecting hydrarthrosis of the knee. J Rheumatol 1999;26:2681-3.

8 Cellerini M, Salti S, Trapani S, D’Elia G, Falcini F, Villari N. Correlation between clinical and ultrasound assessment of the knee in children with monoarticular or pauci-articular juvenile rheumatoid arthritis. Pediatr Radiol 1999;29: 117-23

9 Lehtinen A, Taavitsainen M, Leirisalo-Repo M. Sonographic analysis of enthesopathy in the lower extremities of patients with spondylarthropathy. Clin Exp Rheumatol 1994;12:143-8.

10 Balint PV, Kane D, Hunter JA, Mclnnes IB, Field M, Sturrock RD. A comparison of ultrasound-guided with conventional guided joint aspiration in rheumatology practice-a pilot study. J Rheumatol 2002;29:2209-13.

11 Handy JR. Popliteal cysts in adults: a review. Semin Arthritis Rheum 2001;31:108-18

12 Balint PV, Sturrock RD. Inflamed retrocalcaneal bursa and Achilles tendonitis in psoriatic arthritis demonstrated by ultrasonography. Ann Rheum Dis 2000;59:931-3.

13 Cunnane G, Brophy DP, Gibney RG, Fitz Gerald O. Diagnosis and treatment of heel pain in chronic inflammatory arthritis using ultrasound. Semin Arthritis Rheum 1996;25:383-9. 
14 Brophy DP, Cunnane G, Fitzgerald O, Gibney RG. Technical report: ultrasound guidance for injection of soft tissue lesions around the heel in chronic inflammatory arthritis. Clin Radiol 1995;50:120-2.

15 Shrier I, Matheson GO, Kohl HW 3rd. chilles tendonitis: are corticosteroid iniections useful or harmful? Clin J Sport Med 1996:6:245-50.

16 Waterston SW, Maffulli N, Ewen SW. Subcutaneous rupture of the Achilles tendon: basic science and some aspects of clinical practice. Br J Sports Med 1997;31:285-98.
17 Bianchi S, Martinoli C, Abdelwahab IF, Derchi LE, Damiani S. Sonographic evaluation of tears of the gastrocnemius medial head ("tennis leg") [see comments]. J Ultrasound Med 1998;17:157-62.

18 Gaulrapp H. ["Tennis leg": ultrasound differential diagnosis and follow-up.] Sportverletz Sportschaden 1999:13:53-8.

19 Karim Z, Wakefield RJ, Conaghan PG, Lawson CA, Goh E, Quinn MA, et al. The impact of ultrasonography on diagnosis and management of patients with musculoskeletal conditions. Arthritis Rheum 2001;44:2932-3.

\section{UNUSUAL AND MEMORABLE}

\section{Case Number 28: Rheumatoid arthritis and congenitally short fourth metacarpals}

Series editor: Gary D Wright

A 53 year old man had a long history of rheumatoid arthritis (RA) necessitating hip, knee and elbow replacements. Hand deformity and pain progressed (right worse), particularly at the metacarpophalangeal (MCP) joints. However, he had congenital short fourth metacarpals and fourth MCP joints which were clinically and radiologically sound. He underwent prosthetic replacements of the right $2 \mathrm{nd} / 3 \mathrm{rd} / 5$ th MCP joints (fig $1 \mathrm{~B}$ ). A similar picture (preoperative) was seen in the left hand (fig $1 \mathrm{~A}$ ).

Radiographs may underestimate the severity of RA changes, but it is interesting to speculate why an MCP joint of a short metacarpal might be protected against degeneration. There might be an associated MCP joint anomaly, making it less prone to erosive change. However, we suggest
Ann Rheum Dis 2004;63:14. doi: 10.1136/ard.2003.012500

that MCP joint biomechanics are important-not only are flexor and extensor functions altered but also the fourth MCP joint is splinted by the neck/head of the 3rd and 5th metacarpals, preventing valgus/varus deviation.

Although RA is appropriately considered an inflammatory condition, these pictures emphasise the importance of mechanical factors in joint failure.

J S Huntley, C R Howie

Department of Orthopaedics, New Royal Infirmary, Old Dalkeith Road, Edinburgh EH16 4SU, UK

Correspondence to: Dr J S Huntley; jimhuntley@doctors.org.uk
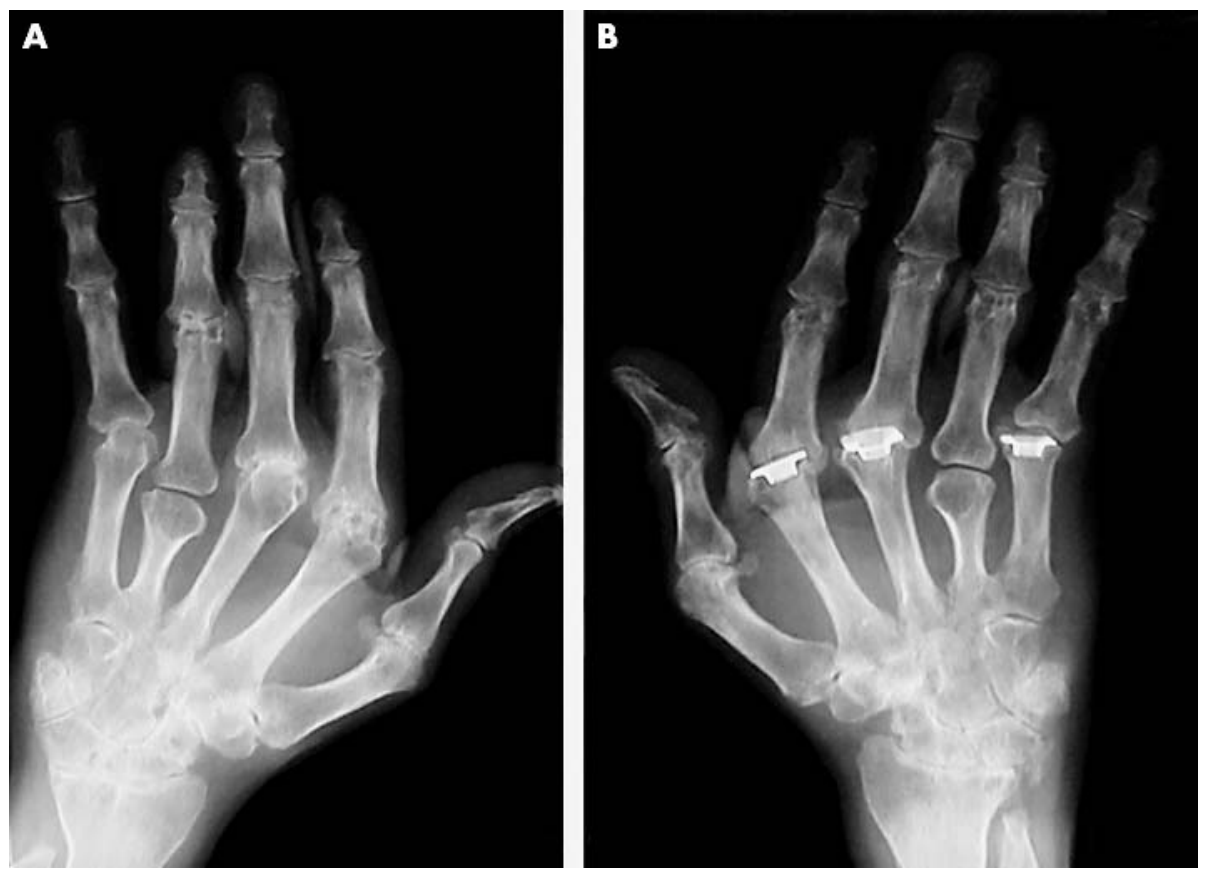

Figure 1 Posteroanterior views of the left (A) and right (B) hands. 